\title{
Analysis of Electric-Field Stimulated Time-resolved X-ray Crystallography Data
}

\author{
Doeke R. Hekstra ${ }^{1}$, Bo Ram Lee ${ }^{2}$, Kevin M. Dalton ${ }^{1}$, Rama Ranganathan ${ }^{2}$
}

1. Department of Molecular and Cellular Biology, and School of Engineering and Applied Sciences, Harvard University; 52 Oxford Street, Cambridge, MA 02138; doeke_hekstra@ harvard.edu

2. The Center for the Physics of Evolving Systems, Department of Biochemistry and Molecular Biology, and The Institute for Molecular Engineering, University of Chicago

The concerted motions of amino acid residues in proteins play important roles in enzyme catalysis, transport and signal transmission across membranes, ligand binding, and allosteric control of protein function. To study such motions, and the mechanical properties of proteins which support them, we developed Electric-Field Stimulated Time-resolved X-ray Crystallography, or EF-X [1].

In EF-X, we apply strong nanosecond-to-microsecond electric field pulses to proteins, and observe the induced structural changes by time-resolved X-ray crystallography. The combination of knowable, controllable patterns of force, and the direct observation of resulting atomic displacements provides a path towards systematic elucidation of the mechanical basis of protein function and allosteric control.

I will describe experimental and analytical advances in order to sensitively obtain structural signals from datasets obtained using short X-ray pulses at both synchrotrons and X-ray free electron lasers.

\section{References}

[1] Hekstra, D. R. et al. Nature 540, 400-405 (2016). 